ORIGINAL ARTICLE

\title{
Emergency department patients' opinions of screening for intimate partner violence among women
}

\author{
K F Hurley, T Brown-Maher, S G Campbell, T Wallace, R Venugopal, D Baggs
}

Emerg Med J 2005;22:97-98. doi: 10.1136/emj.2002.002626

See end of article for authors' affiliations .....................

Correspondence to: Dr K F Hurley, Department of Emergency Medicine, Dalhousie University, 351 Bethune, VG Site, QEII Health Sciences Centre, 1278 Tower Road, Halifax, NS B3H 2 Y9 Canada; kfhurley@dal.ca

Accepted for publication 10 June 2003
Background: Universal screening for intimate partner violence (IPV) in the emergency department (ED) has been advocated by many medical institutions. Policies implemented for IPV screening have met with numerous obstacles. One such obstacle is the perception by emergency personnel that patients might be offended by such screening if they presented to the ED for problems unrelated to trauma.

Objectives: To assess opinions of adult ED patients regarding a policy of universal IPV screening for women presenting to the ED.

Methods: This study was conducted in EDs in Halifax, Nova Scotia, and St John's, Newfoundland. Patients were questioned as to whether it was appropriate for all women to be asked if they had experienced violent or threatening behaviour from someone close to them. Patients in significant pain or in extremis were not approached.

Results: The data consist of a convenience sample of 514 adult ED patients, aged 16-95 years. Two $(0.4 \%)$ were excluded from the analysis. Of 512 analysed, $442(86.0 \%)$ answered "yes" to the question, $53(10.3 \%)$ answered "no", $17(3.3 \%)$ had no opinion. There were no significant differences between the proportion of "yes" and "no" answers in the male and female groups.

Conclusion: Universal screening for IPV of adult female patients presenting to the ED was supported by most patients. Patient objections should not be seen as a reason to withhold questioning on this issue. l ntimate partner violence (IPV) is defined as a pattern of assault or controlling behaviour against one partner by the other, including physical, sexual, and psychological abuse. ${ }^{1}$ Eighty eight per cent of spousal violence victims in Canada are female. ${ }^{12}$ IPV victims are at higher risk for chronic pain, drug and alcohol abuse, depression, and suicide attempts, making IPV a significant public health issue, particularly in women. ${ }^{3}$

In Canada and the United States, $27 \%-30 \%$ of women who seek treatment in the emergency room are victims of IPV..$^{3-6}$ Because the emergency department (ED) is an entry point into the healthcare system for victims, it has been suggested that screening of all women that present to the ED may improve detection and treatment of abuse victims. ${ }^{367}$

Universal screening for IPV using direct questioning has been successfully used by several researchers, yet departmental screening protocols have been difficult to implement. ${ }^{5-8}$ Reluctance of emergency personnel may stem from concerns about time constraints, lack of adequate community referral agencies, a sense of frustration about victims that do not leave their partners or seek help, and perception by emergency personnel that patients might be offended by screening if they present to the ED for problems unrelated to trauma. ${ }^{159}$ This study was undertaken to determine if a significant proportion of the population that visit the ED would approve of such a universal screening programme, and whether opinion varied with age or sex.

\section{METHODS}

This study was conducted in EDs at The Queen Elizabeth II Health Sciences Centre in Halifax, NS (QE II), and the Health Sciences Centre in St John's, NF (HSC), both tertiary care centres with an ED census of 75000 and 42000 visits a year, respectively.

Data collection occurred in 2000 at QE II (over one year) and in May 2001 at HSC. Patients were approached during both daytime and night time hours, although time of day was not recorded for data analysis. All aspects of this study were reviewed and approved by human investigation and ethics committees at the respective institutions.

Patient consent was obtained before surveying and no identifying data were recorded. On an a priori basis, a sample size of 500 was chosen. After obtaining verbal consent, the subject was read the following standardised script and asked to answer "yes", "no", or "no opinion":

\begin{abstract}
"We know that women often experience abuse or violence committed by people close to them. Emergency departments are often the only place where these women can be identified and offered help. Right now we do not currently ask every patient about abuse and violence. We would like your opinion as to whether it is appropriate for the doctor to privately ask all women coming to the emergency department if they have experienced violent or threatening behaviour from someone close to them."
\end{abstract}

Patients recorded their age, sex, and responses privately and completed surveys were placed into a sealed envelope. Patients were not asked to disclose personal experiences with violence.

Subjects included non-critically ill patients, aged 16-95 years who presented to the ED during specified data collection time frames. All potentially eligible patients in the waiting room were approached individually during these time frames. Subjects who refused or were in distress were not included. Medical students administering the survey did not interfere with the active medical care of patients and were not aware of the presenting complaint.

Data were entered into SPSS and analysed using descriptive statistical methods. A $\chi^{2}$ analysis of the relation between

Abbreviations: IPV, intimate partner violence; $E D$, emergency department 
Table 1 Patient opinion on universal IPV screening

\begin{tabular}{llllll}
\hline & Approved & Disapproved & No opinion & Excluded & Total \\
\hline Male & 179 & 21 & 8 & 2 & 210 \\
Female 263 & 32 & 9 & 0 & 304 \\
Total 442 & 53 & 17 & 2 & 514 \\
\hline
\end{tabular}

opinion and sex was performed. A $t$ test was used to determine any association between opinion and age. An $\alpha$ level of 0.05 was used.

\section{RESULTS}

In total, 514 undifferentiated ED patients, (mean age 39.4 years) were surveyed; 250 at QE II and 264 at HSC. No language barriers were identified. Altogether 304 (59.1\%) were female and $210(40.9 \%)$ were male. A total of 442 $(86.0 \%)$ answered "yes" to the survey question, 53 (10.3\%) answered "no", and 17 (3.3\%) had no opinion (table 1). Two $(0.4 \%)$ subjects were excluded; one became volatile and refused to answer questions while the second was found incapable of responding because of medical reasons.

A $\chi^{2}$ analysis was completed to review the relation between opinion and sex. There were no significant differences between the proportion of "yes" and "no" answers in the male and female groups $(p>0.05)$. Because the data followed a normal distribution, a $t$ test was used showing no significant differences between age and opinion when sex was reviewed separately or combined $(\mathrm{p}>0.05)$.

There were no significant differences found between the data collected at each centre.

\section{DISCUSSION}

The medical community has recognised IPV as a significant public health issue. Female victims who present to the ED, often have non-traumatic presenting complaints and frequently choose not to disclose IPV unless asked directly by a physician or other healthcare professional in a safe and private setting. ${ }^{10}{ }^{11}$ A 1997 survey found that of all domestic violence victims, $36 \%$ of women would only divulge domestic violence if asked directly. ${ }^{11}$ By not inquiring about the risk of domestic violence, the physician overlooks a causative factor in the patient's illness and an opportunity to prevent further violence.

Many barriers to intervention have been described. Our study shows that universal screening of female patients for IPV is supported by $86 \%$ of patients, with no differences in opinion with relation to age or sex. Patient objection or offence should not be seen as a reason to withhold questioning on this issue, a finding that is compatible with other studies. ${ }^{12}{ }^{13}$ We acknowledge that selection bias must be considered in this study as the only eligibility criteria used were patients being non-critically ill, and presentation to the ED during the study period. We were unable to determine the proportion of all eligible patients that were approached or their presenting complaint. Although an effort was made to ensure that all times of the day were represented, these data were not recorded and distribution of patients interviewed during daytime and night time hours cannot be determined. This may represent a source of further selection bias.

Response bias is also an important issue because patients had to be verbally asked a "socially charged" question. Verbal questioning was necessary because of a high rate of illiteracy (one in four Canadians are functionally illiterate). ${ }^{14}$ We attempted to minimise response bias within this study by giving patients the opportunity to complete surveys in private.

Refusals to participate were not recorded. This makes it impossible to generalise non-responders to the population at large or to rule out a selection bias between responders and non-responders.

Our survey questioned whether ED patrons believed all female patients should be screened for IPV. Research shows an IPV prevalence rate of $13 \%$ inflicted by women upon men. ${ }^{15}$ Restricting the screening group to women only, may allow abuse of elderly people, disabled persons, and men to go undetected.

To direct medical curriculum and ED protocol development, further research into current attitudinal barriers regarding domestic violence is necessary, including the impact of patient characteristics such as sexual orientation, socioeconomic status, and cultural beliefs.

In conclusion, universal screening for IPV of adult females presenting to the ED was supported by most patients. Fear of patient objections or offence is not a valid reason to withhold screening for IPV.

\section{Authors' affiliations}

K F Hurley, S G Campbell, T Wallace, Department of Emergency

Medicine, Dalhousie University, Halifax, Canada

T Brown-Maher, Department of Dermatology, McGill University,

Montreal, Quebec, Canada

D Baggs, Department of Emergency Medicine, Memorial University of Newfoundland, Canada

R Venugopal, Department of Emergency Medicine, McGill University

\section{REFERENCES}

1 Little K. Screening for domestic violence: identifying, assisting, and empowering adult victims of abuse. Postgrad Med 2000;108:135-41.

2 Canadian Centre for Justice Statistics, Statistics Canada. Family violence in Canada: a statistical profile July 2000. http://stcwww.statcan.ca/english/ freepub/85-224-XIE/0000085-224-XIE.pdf (accessed May 2002).

3 Swanson L. Abuse against women a public health issue: MD. Can Med Assoc J $2000 ; 162: 848$.

4 Statistics Canada. Family violence in Canada. Ottawa: Statistics Canada, 1994.

5 Morrison LJ, Allan R, Grunfeld A. Improving the emergency department detection rate of domestic violence using direct questioning. J Emerg Med 2000; 19:117-24

6 Feldhaus KM, Koziol-McLain J, Amsbury HL, et al. Accuracy of 3 brief screening questions for detecting partner violence in the emergency department. JAMA 1997;277:1357-61.

7 McLeer SV, Anwar R. A study of battered women presenting in an emergency department. Am J Public Health 1989;79:65-6.

8 Brown JB, Lent B, Brett PJ, et al. Development of the woman abuse screening tool for use in family practice. Fam Med 1996;28:422-8.

9 Garimella R, Plixhta SB, Houseman C, et al. Physician beliefs about victims of spouse abuse and about the physician role. Journal of Womens's Health and Gender-Based Medicine 2000;9:405-11.

10 Abbott J, Johnson R, Koziol-McLain J, et al. Domestic violence against women: incidence and prevalence in an emergency department population. JAMA 1995;273:1763-7

11 Hayden SR, Barton ED, Hayden M. Domestic violence in the emergency department: how do women prefer to disclose and discuss the issues? J Emerg Med 1997; 15:447-51.

12 Freidman LS, Samet JH, Robers MS, et al. Inquiry about victimization experiences: A survey of patient preferences and physician practices. Arch Intern Med 1992;153:1186-90.

13 Roberts GL, O'Toole BI, Lawrence JM, et al. Domestic violence victims in a hospital emergency department. Med J Aust 1993;159:307-10.

14 Newfoundland and Labrador Laubach Literacy Council. Literacy is for life! May 2002. http://www.nald.ca/Province/Nfld/nlllc/life.html.

15 Mechem CC, Shofer FS, Reinhard SS, et al. History of domestic violence among male patients presenting to an urban emergency department. Acad Emerg Med 1999;6:786-91. 